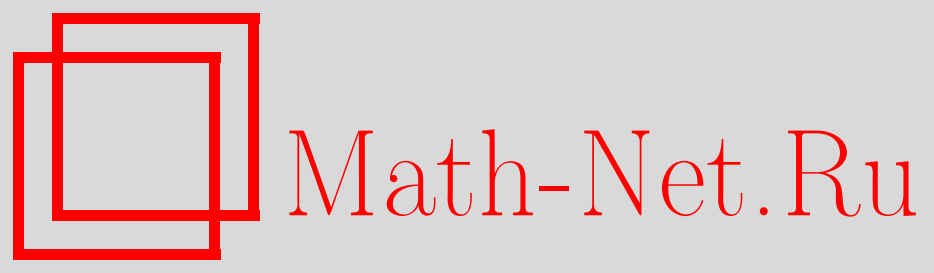

О. П. Орлов, Н. Ю. Пасынков, Распределения межрекордных наполнений, Дискрет. матем., 2015, том 27, выпуск 3, 56-73

DOI: https://doi.org/10.4213/dm1235

Использование Общероссийского математического портала Math-Net.Ru подразумевает, что вы прочитали и согласны с пользовательским соглашением http://www . mathnet.ru/rus/agreement

Параметры загрузки:

IP : 54.198 .55 .26

26 апреля 2023 г., 14:06:14

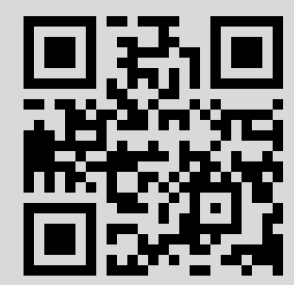




\title{
Распределения межрекордных наполнений
}

\author{
() 2015 г. О. П. Орлов* , Н. Ю. Пасынков**
}

В последовательности независимых положительных случайных величин с одной и той же непрерывной функцией распределения выделяется монотонная подпоследовательность рекордных значений. Соответствующая им последовательность рекордных моментов разбивает исходную последовательность на межрекордные интервалы. Интервалу между $i$-м и $(i+1)$-м рекордными моментами сопоставляются числа $\alpha_{i}^{j}(j=1, \ldots, i)$ - случайные величины, значения которых попадают между $(j-1)$-м и $j$-м рекордами. Получены явные формулы для совместных распределений случайных величин $\alpha_{i}^{j}, 1 \leqslant j \leqslant i \leqslant n$, доказаны предельные теоремы для распределений $\alpha_{i}^{j}$ при $i-j \rightarrow \infty$.

Ключевые слова: независимые случайные величины, рекорды, рекордные моменты, явные формулы для распределений, предельные теоремы.

\section{Введение}

Пусть $X_{1}, X_{2}, \ldots$ - последовательность независимых одинаково распределенных положительных случайных величин с общей функцией распределения $F(x)(x \in \mathbb{R})$, которая всюду далее предполагается непрерывной. Определим рекордные значения в этой последовательности и моменты их появления. Первой рекордной величиной является $X_{1}$. Положим $L(0)=0, L(1)=1$. Если $m-$ наименьшее натуральное число, для которого $X_{m}>X_{1}$, то назовем $X_{m}$ вторым рекордом, a $m-$ вторым рекордным моментом, и обозначим его $L(2)$. Продолжая этот процесс, получим последовательность рекордных моментов $L(n), n \geqslant 1$, и последовательность рекордов $X_{L(n)}, n \geqslant 1$. Элемент последовательности $X_{L(n)}$ является наибольшим в выборке $X_{1}, X_{2}, \ldots, X_{n}, \ldots, X_{L(n)}$, т. е. действительно является $n$-м рекордом, а $L(n)-n$-м рекордным моментом.

Формально последовательность $\{L(n)\}$ определяется равенствами

$$
L(1)=1 ; L(n)=\min \left\{m \mid X_{m}>X_{L(n-1)}\right\}, n>1 .
$$

В этой работе изучается структура последовательности $\left\{X_{n}\right\}$ в межрекордных интервалах $(L(n), L(n+1))$ длины $\Delta(n)=L(n+1)-L(n), n \geqslant 1$. Для произвольного фиксированного натурального числа $i$ рассмотрим случайные величины $X_{L(i)+1}, X_{L(i)+2}, \ldots, X_{L(i+1)-1}$. Каждая из них попадает в один из непересекающихся интервалов $\left(0, X_{L(1)}\right],\left(X_{L(1)}, X_{L(2)}\right], \ldots,\left(X_{L(i-1)}, X_{L(i)}\right]$. Обозначим количество

* Место работы: МГУ им. М. В. Ломоносова, e-mail: olegorlov92@gmail.com

** Место работы: МГУ им. М. В. Ломоносова, e-mail: nickpasynkov@gmail.com 
случайных величин, попавших в первый интервал, через $\alpha_{i}^{1}$, количество случайных величин, попавших во второй интервал, через $\alpha_{i}^{2}$ и так далее. Последовательность $\alpha_{i}^{j}, 1 \leqslant j \leqslant i$, назовем межрекордными наполнениями. В общем случае

$$
\alpha_{i}^{j}=\sum_{l=L(i)+1}^{L(i+1)-1} I\left\{X_{L(j-1)}<X_{l} \leqslant X_{L(j)}\right\}, 1 \leqslant j \leqslant i,
$$

т. е. $\alpha_{i}^{j}$ равно количеству случайных величин с индексами между $L(i)$ и $L(i+1)$ и попавших в промежуток между рекордами $X_{L(j-1)}$ и $X_{L(j)}$. Случайные величины $\alpha_{i}^{j}$ естественно связаны с величинами $\Delta(i)$ и $L(n)$ :

$$
\begin{gathered}
\Delta(i)=L(i+1)-L(i)=\sum_{j=1}^{i} \alpha_{i}^{j}+1, \\
L(n)=1+\sum_{i=1}^{n-1} \Delta(i)=n+\sum_{i=1}^{n-1} \sum_{j=1}^{i} \alpha_{i}^{j} .
\end{gathered}
$$

В настоящей работе найдено совместное распределение межрекордных наполнений, частично описана вероятностная структура этого распределения, доказан ряд одномерных предельных соотношений для межрекордных наполнений.

\section{1. Формулировки результатов}

Теорема 1. При любом $k \in \mathbb{N}$ совместное распределение случайных величин $\alpha_{i}^{j}, 1 \leqslant j \leqslant i \leqslant k$, находится по формуле

$$
\mathrm{P}\left\{\bigcap_{1 \leqslant j \leqslant i \leqslant k}\left\{\alpha_{i}^{j}=a_{i}^{j}\right\}\right\}=\frac{\prod_{i=1}^{k} A_{i} ! \cdot \prod_{j=1}^{k} A^{j} !}{N ! \cdot \prod_{1 \leqslant j \leqslant i \leqslant k} a_{i}^{j} !}, \quad a_{i}^{j} \in\{0,1, \ldots\}, 1 \leqslant j \leqslant i \leqslant k,
$$

əде $A_{i}=\sum_{j=1}^{i} a_{i}^{j}, A^{j}=\sum_{i=j}^{k} a_{i}^{j}, N=A_{1}+\ldots+A_{k}+k+1=A^{1}+\ldots+A^{k}+k+1$.

Замечание. В доказательствах теорем 1-4 фактически вместо условия независимости случайных величин $\left\{X_{n}, n \in \mathbb{N}\right\}$ используется лишь свойство их перестановочности (т. е. для любого $N \geqslant 2$ и любой перестановки $\sigma=\left(\sigma_{1}, \ldots, \sigma_{N}\right) \in S_{N}$ распределения $\left(X_{1}, \ldots, X_{N}\right)$ и $\left(X_{\sigma_{1}}, \ldots, X_{\sigma_{N}}\right)$ одинаковы $)$.

Следствие 1 и теоремы 2 - 4 описывают вероятностные свойства межрекордных наполнений.

Следствие 1. Для любых $k, r \in \mathbb{N} u$ любых таких натуралъных $i_{1}, \ldots, i_{r}$, $j_{1}, \ldots, j_{r}$, что $i_{1} \leqslant i_{2} \leqslant \ldots \leqslant i_{r} \leqslant k u j_{1} \leqslant i_{1}, \ldots, j_{r} \leqslant i_{r}$, распределения набоpos

$$
\left(\alpha_{i_{1}}^{j_{1}}, \ldots, \alpha_{i_{r}}^{j_{r}}\right) u\left(\alpha_{k-j_{1}+1}^{k-i_{1}+1}, \ldots, \alpha_{k-j_{r}+1}^{k-i_{r}+1}\right)
$$


одинаковъ (симметричность относительно диагонали $\left.\alpha_{k}^{1}, \alpha_{k-1}^{2}, \alpha_{k-2}^{3}, \ldots\right)$. В частности, $\alpha_{i}^{j}$ и $\alpha_{i-j+1}^{1}$ одинаково распределень (т.е. одномерные распределения межрекордных наполнений исчерпываются распределениями случайных величин $\left.\alpha_{k}^{1}, k \in \mathbb{N}\right)$.

Пусть $m, n \in \mathbb{N}$. Обозначим событие, состоящее в том, что случайные величины $\alpha_{i}^{j}$ принимают заданные значения $a_{i}^{j}, m \leqslant j \leqslant i \leqslant n$, через $B_{m}^{n}=$ $\left\{\cap_{m \leqslant j \leqslant i \leqslant n}\left\{\alpha_{i}^{j}=a_{i}^{j}\right\}\right\}$.

Теорема 2. Для любых $m, n \in \mathbb{N}$ и любых чисел $a_{i}^{j} \in \mathbb{N}_{0}$ справедливо равенство

$$
\mathrm{P}\left\{\bigcap_{1 \leqslant j \leqslant i \leqslant n}\left\{\alpha_{i}^{j}=a_{i}^{j}\right\}\right\}=\mathrm{P}\left\{\bigcap_{1 \leqslant j \leqslant i \leqslant n}\left\{\alpha_{i+m}^{j+m}=a_{i}^{j}\right\}\right\} .
$$

Таким образом, распределение любого набора значений $\alpha_{i}^{j}$ не изменяется при одновременном увеличении всех верхних и нижних индексов на одно и то же число.

Теорема 3. Для любых $m, n \in \mathbb{N}$ и любых чисел $a_{i}^{j} \in \mathbb{N}_{0}$ справедливо равенство

$$
\mathrm{P}\left(B_{1}^{m} \cap B_{m+1}^{m+n}\right)=\mathrm{P}\left(B_{1}^{m}\right) \cdot \mathrm{P}\left(B_{m+1}^{m+n}\right) .
$$

В частности, для любых $k, r, t \in \mathbb{N}$ и любых таких натуральных чисел $i_{1}, \ldots, i_{r+t}, j_{1}, \ldots, j_{r+t}$, что $i_{1} \leqslant \ldots \leqslant i_{r} \leqslant k<i_{r+1} \leqslant \ldots \leqslant i_{r+t}$ и $j_{1} \leqslant i_{1}, \ldots, j_{r} \leqslant$ $i_{r}, k<j_{r+1} \leqslant i_{r+1}, \ldots, k<j_{r+t} \leqslant i_{r+t}$ случайные векторы $\left(\alpha_{i_{1}}^{j_{1}}, \ldots, \alpha_{i_{r}}^{j_{r}}\right)$ и $\left(\alpha_{i_{r+1}}^{j_{r+1}}, \ldots, \alpha_{i_{r+t}}^{j_{r+t}}\right)$ независимы.

Теорема 4. Для любых $m, n, q \in \mathbb{N}$ и любых чисел $a_{i}^{j} \in \mathbb{N}_{0}$ справедливо равенство

$$
\mathrm{P}\left(B_{1}^{m+q} \cap B_{m+1}^{m+q+n} \mid B_{m+1}^{m+q}\right)=\mathrm{P}\left(B_{1}^{m+q} \mid B_{m+1}^{m+q}\right) \cdot \mathrm{P}\left(B_{m+1}^{m+q+n} \mid B_{m+1}^{m+q}\right) .
$$

Таким образом, векторы $\left(\alpha_{i}^{j}, 1 \leqslant j \leqslant i \leqslant m+q\right)$ и $\left(\alpha_{i}^{j}, m+1 \leqslant j \leqslant i \leqslant m+q+n\right)$ условно независимы при условии $\left(\alpha_{i}^{j}, m+1 \leqslant j \leqslant i \leqslant m+q\right)$.

Теорема 5 даёт интегральное представление одномерного распределения межрекордного наполнения.

Теорема 5. Для любого натурального $m$ u таких натуральнъх $i, j$, что $i>j$, распределение случайной величины $\alpha_{i}^{j}$ записъвается в виде

$$
\mathrm{P}\left(\alpha_{i}^{j} \geqslant m\right)=\int_{0}^{\infty} \frac{x^{i-j-1}}{(i-j-1) !} e^{-x} \int_{0}^{\infty}\left[1-\frac{e^{-x}}{e^{y}+e^{-x}-1}\right]^{m} e^{-y} d y d x
$$

Теоремы 6 и 7 описывают асимптотические свойства одномерного распределения $\alpha_{i}^{j}$. 
Теорема 6. Справедливы пределъные соотношения

$$
\begin{gathered}
\frac{\ln \alpha_{i}^{j}-(i-j+1)}{\sqrt{i-j+1}} \frac{D}{i-j \rightarrow \infty} N(0,1), \quad \lim _{i-j \rightarrow \infty} \frac{\ln \alpha_{i}^{j}}{i-j+1} \stackrel{n . \mu .}{=} 1, \\
\limsup _{i-j \rightarrow \infty} \frac{\ln \alpha_{i}^{j}-(i-j+1)}{\sqrt{2(i-j+1) \ln \ln (i-j+1)}} \stackrel{\text { n... }}{=} 1, \\
\liminf _{i-j \rightarrow \infty} \frac{\ln \alpha_{i}^{j}-(i-j+1)}{\sqrt{2(i-j+1) \ln \ln (i-j+1)}} \stackrel{\text { n.H. }}{=}-1 .
\end{gathered}
$$

Теорема 7. Имеет место сходимость распределений

$$
\mathrm{P}\left\{\frac{\alpha_{k}^{1}}{\Delta(k)}>x\right\} \underset{k \rightarrow \infty}{\longrightarrow} 1-x, \quad 0 \leqslant x \leqslant 1 .
$$

\section{2. Свойства совместного распределения $\alpha_{i}^{j}$. Доказа- тельства теорем 1-4}

Приведем доказательство теоремы 1 , основанное только на том, что случайные величины $X_{1}, X_{2}, \ldots$ перестановочны и имеют непрерывную функцию распределения.

Доказательство теоремы 1. Пусть $a_{i}^{j}(1 \leqslant j \leqslant i \leqslant k)$ - любой набор целых неотрицательных чисел, величины $A_{i}, A^{j}, N$ определяются так же, как в условии теоремы 1 . Если $X_{1}, \ldots, X_{N}$ - перестановочные случайные величины, с вероятностью 1 принимающие различные значения, то вероятность события $B_{1}^{k}=\left\{\alpha_{i}^{j}=\right.$ $\left.a_{i}^{j}, 1 \leqslant j \leqslant i \leqslant k\right\}$ равна доле таких перестановок $\pi=\left(\pi_{1}, \ldots, \pi_{N}\right) \in S_{N}$, что событие $B_{1}^{k}$ выполняется для величин $\alpha_{i}^{j}(\pi)$, построенных по последовательности чисел $\pi_{1}, \ldots, \pi_{N}$, принимающих значения $1, \ldots, N$. Для этой последовательности условие $B_{1}^{k}$ означает, что (при $A_{0}=A^{0}=0$ )

$$
\begin{gathered}
\pi_{A_{0}+\ldots+A_{j-1}+j}=A^{0}+\ldots+A^{j}+j>\max \left\{\pi_{1}, \ldots, \pi_{A_{0}+\ldots+A_{j-1}+j-1}\right\}, j=1, \ldots, k, \\
\pi_{A_{0}+\ldots+A_{k}+k+1}=A^{0}+\ldots+A^{k}+k+1=N
\end{gathered}
$$

и

$$
\left|\left(\bigcup_{m=A_{0}+\ldots+A_{i-1}+i+1}^{A_{0}+\ldots+A_{i}+i}\left\{\pi_{m}\right\}\right) \bigcap\left\{j+\sum_{m=0}^{j-1} A^{m}, \ldots, j-1+\sum_{m=0}^{j} A^{m}\right\}\right|=a_{i}^{j},
$$

$1 \leqslant j \leqslant i \leqslant k$. Количество вариантов разбить множество $\left\{\pi_{m}, A_{0}+\ldots+A_{i-1}+i+1 \leqslant\right.$ $\left.m \leqslant A_{0}+\ldots+A_{i}+i\right\}$ на подмножества из $a_{i}^{1}, \ldots, a_{i}^{i}$ элементов соответственно равно $\frac{A_{i} !}{a_{i}^{1} ! \ldots a_{i}^{i} !}$. После того, как построены такие разбиения на подмножества для всех $i=1, \ldots, k$, нужно учесть, что количество способов перестановок элементов множеств

$$
\left\{A^{0}+\ldots+A^{j-1}+j, \ldots, A^{0}+\ldots+A^{j}+j-1\right\}, j=1, \ldots, k
$$


равно $A^{1} ! \ldots A^{k}$ !. Поэтому количество таких перестановок $\pi$, что событие $B_{1}^{k}$ выполняется для величин $\alpha_{i}^{j}(\pi)$, равно

$$
\frac{A_{1} !}{a_{1}^{1} !} \cdot \frac{A_{2} !}{a_{2}^{1} ! a_{2}^{2} !} \cdot \ldots \cdot \frac{A_{k} !}{a_{k}^{1} ! \cdot \ldots \cdot a_{k}^{k} !} \cdot A^{1} ! \cdot A^{2} ! \cdot \ldots \cdot A^{k} !=\frac{\prod_{i=1}^{k} A_{i} ! \cdot \prod_{j=1}^{k} A^{j} !}{\prod_{1 \leqslant j \leqslant i \leqslant k} a_{i}^{j} !}
$$

Следовательно,

$$
\mathrm{P}\left(B_{1}^{k}\right)=\frac{\prod_{i=1}^{k} A_{i} ! \cdot \prod_{j=1}^{k} A^{j !}}{\prod_{1 \leqslant j \leqslant i \leqslant k} a_{i}^{j} ! \cdot N !}
$$

Теорема 1 доказана.

Следствие 2. Пусть $\eta_{k}^{1}=\alpha_{1}^{1}+\alpha_{2}^{1}+\ldots+\alpha_{k}^{1}-$ количество случайных величин $X_{n}$ до $(k+1)$-го рекорда, которые меньше $X_{1}$. Согласно следствию 1 случайная величина $\eta_{k}^{1}$ имеет такое же распределение, как $\Delta(k)$ - 1. Поэтому все теоремы для $\Delta(k)$ переносятся на случайные величины $\eta_{k}^{1}$.

Доказательство теоремы 2. По следствию 1

$$
\mathrm{P}\left\{\bigcap_{1 \leqslant j \leqslant i \leqslant n}\left[\alpha_{i+m}^{j+m}=a_{i}^{j}\right]\right\}=\mathrm{P}\left\{\bigcap_{1 \leqslant j \leqslant i \leqslant n}\left[\alpha_{n-j+1}^{n-i+1}=a_{i}^{j}\right]\right\}
$$

(здесь мы воспользовались симметричностью относительно диагонали $\left.\left\{\alpha_{m+n}^{1}, \alpha_{m+n-1}^{2}, \alpha_{m+n-2}^{3}, \ldots\right\}\right)$. Сделаем замену $s=n-i+1, t=n-j+1$ :

$$
\mathrm{P}\left\{\bigcap_{1 \leqslant j \leqslant i \leqslant n}\left[\alpha_{n-j+1}^{n-i+1}=a_{i}^{j}\right]\right\}=\mathrm{P}\left\{\bigcap_{1 \leqslant s \leqslant t \leqslant n}\left[\alpha_{t}^{s}=a_{n-s+1}^{n-t+1}\right]\right\} .
$$

Воспользуемся теперь симметричностью относительно диагонали $\left\{\alpha_{n}^{1}, \alpha_{n-1}^{2}, \alpha_{n-2}^{3}, \ldots\right\}$ и сделаем обратную замену $i=n-s+1, j=n-t+1$. Получим

$$
\begin{gathered}
\mathrm{P}\left\{\bigcap_{1 \leqslant s \leqslant t \leqslant n}\left[\alpha_{t}^{s}=a_{n-s+1}^{n-t+1}\right]\right\}=\mathrm{P}\left\{\bigcap_{1 \leqslant s \leqslant t \leqslant n}\left[\alpha_{n-s+1}^{n-t+1}=a_{n-s+1}^{n-t+1}\right]\right\}= \\
=\mathrm{P}\left\{\bigcap_{1 \leqslant j \leqslant i \leqslant n}\left[\alpha_{i}^{j}=a_{i}^{j}\right]\right\} .
\end{gathered}
$$

Теорема 2 доказана.

Доказательство теорем 3, 4. Пусть $a_{i}^{j}(1 \leqslant j \leqslant i \leqslant m+q+n)$ - любой набор целых неотрицательных чисел, величины $A_{i}, A^{j}$ определяются так же, как в условии теоремы 1. Определим величины $A^{\prime j}=\sum_{i=j}^{m+q} a_{i}^{j}, 1 \leqslant j \leqslant m+q, A_{i}^{\prime \prime}=\sum_{j=m+1}^{i+m+q} a_{i+m+q}^{j}$, $1 \leqslant i \leqslant n\left(A_{0}^{\prime \prime}=A^{\prime 0}=0\right)$. Обозначим $N_{1}=A_{1}+\ldots+A_{m+q}+m+q+1, N_{2}=$ 
$A^{m+1}+\ldots+A^{m+q+n}+q+n+1, N_{3}=A^{\prime m+1}+\ldots+A^{\prime m+q}+q+1$. По формуле полной вероятности

$$
\mathrm{P}\left(B_{1}^{m+q} \cap B_{m+1}^{m+q+n}\right)=\sum_{N=N_{1}+N_{2}-N_{3}}^{\infty} \mathrm{P}\left(B_{1}^{m+q} \cap B_{m+1}^{m+q+n}, L(m+q+n+1)=N\right) .
$$

Аналогично доказательству теоремы 1 заметим, если $X_{1}, \ldots, X_{N}$ - перестановочные случайные величины, которые с вероятностью 1 принимают различные значения, то вероятность события $B_{1}^{m+q} \cap B_{m+1}^{m+q+n} \cap\{L(m+q+n+1)=N\}$ равна доле таких перестановок $\pi=\left(\pi_{1}, \ldots, \pi_{N}\right) \in S_{N}$, что событие $B_{1}^{m+q} \cap B_{m+1}^{m+q+n} \cap\{L(m+q+n+1)=N\}$ выполняется для величин $\alpha_{i}^{j}(\pi)$, построенных по последовательности чисел $\pi_{1}, \ldots, \pi_{N}$, принимающих значения $1, \ldots, N$. Пусть $\pi_{i}^{\prime}=\pi_{\nu_{i}}$, где

$$
\nu_{1}=N_{1}, \nu_{i}=\min \left\{l>\nu_{i-1}: \pi_{l}>\pi_{N_{1}-A_{m}-\ldots-A_{m+q}-q-1}\right\}, 2 \leqslant i \leqslant N_{2}-N_{3}+1 .
$$

Тогда условие $B_{1}^{m+q} \cap B_{m+1}^{m+q+n} \cap\{L(m+q+n+1)=N\}$ означает, что

$$
\begin{gathered}
\pi_{A_{0}+\ldots+A_{j-1}+j}=A^{0}+\ldots+A^{j}+j>\max \left\{\pi_{1}, \ldots, \pi_{A_{0}+\ldots+A_{j-1}+j-1}\right\} \\
\quad(j=1, \ldots, m+q), \\
\mid\left(\begin{array}{c}
A_{0}+\ldots+A_{i}+i \\
\left.\bigcup_{l=A_{0}+\ldots A_{i-1}+i+1}\left\{\pi_{l}\right\}\right) \bigcap\left\{j+\sum_{m=0}^{j-1} A^{m}, \ldots, j-1+\sum_{m=0}^{j} A^{m}\right\} \mid=a_{i}^{j}
\end{array} \quad(1 \leqslant j \leqslant i \leqslant m+q),\right. \\
\pi_{A_{0}^{\prime \prime}+\ldots+A_{j-1}^{\prime \prime}+j}^{\prime}=A^{0}+\ldots+A^{j+m+q}+j+m+q> \\
>\max \left\{\pi_{1}, \ldots, \pi_{A^{0}+\ldots+A^{j+m+q}+j+m+q-1}\right\}(j=1, \ldots, n), \\
\pi_{A_{0}^{\prime \prime}+\ldots+A_{n}^{\prime \prime}+n+1}^{\prime}=N
\end{gathered}
$$

и

$$
\left|\left(\bigcup_{l=A_{0}^{\prime \prime}+\ldots A_{i-1}^{\prime \prime}+i+1}^{A_{0}^{\prime \prime}+\ldots+A_{i}^{\prime \prime}+i}\left\{\pi_{l}^{\prime}\right\}\right) \bigcap\left\{j+\sum_{m=0}^{j-1} A^{m}, \ldots, j-1+\sum_{m=0}^{j} A^{m}\right\}\right|=a_{i+m+q}^{j}
$$

$(1 \leqslant i \leqslant n, \quad m+1 \leqslant j \leqslant m+q+n, \quad j \leqslant i+m+q)$.

Количество способов выбрать элементы $\nu_{2}, \nu_{3}, \ldots, \nu_{N_{2}-N_{3}}$ из множества $\left\{N_{1}+1, \ldots, N-1\right\}$ равно $C_{N-N_{1}-1}^{N_{2}-N_{3}-1}\left(\pi_{1}^{\prime}=\pi_{N_{1}}, \pi_{N_{2}-N_{3}+1}^{\prime}=\pi_{N}\right)$. Количество вариантов разбить множество $\left\{\pi_{l}, A_{0}+\ldots+A_{s-1}+s+1 \leqslant l \leqslant A_{0}+\ldots+A_{s}+s\right\}$ на подмножества из $a_{s}^{1}, \ldots, a_{s}^{s}$ элементов соответственно равно $\frac{A_{s} !}{a_{s}^{1} ! \ldots a_{s}^{s} !}$. Количество вариантов разбить множество

$$
\left\{\pi_{l}^{\prime}, A_{0}^{\prime \prime}+\ldots+A_{t-1}^{\prime \prime}+t+1 \leqslant l \leqslant A_{0}^{\prime \prime}+\ldots+A_{t}^{\prime \prime}+t\right\}
$$

на подмножества из $a_{t}^{m+1}, \ldots, a_{t}^{t}$ элементов соответственно равно $\frac{A_{t}^{\prime \prime} !}{a_{t}^{m+1} ! \ldots a_{t}^{t} !}$. После того, как такие разбиения на подмножества построены для всех $s=1, \ldots, m+q$ 
и $t=m+q+1, \ldots, m+q+n$, нужно учесть, что количество способов перестановок элементов множеств

$$
\left\{A^{0}+\ldots A^{j-1}+j, \ldots, A^{0}+\ldots+A^{j}+j-1\right\}, j=1, \ldots, m+n,
$$

равно $A^{m+1}$ ! ... $A^{m+q+n}$ !, а количество способов перестановок элементов

$$
\left\{\left(\bigcup_{l=1}^{N_{1}} \pi_{l}\right) \bigcap\left\{A^{0}+\ldots+A^{j-1}+j, \ldots, A^{0}+\ldots+A^{j}+j-1\right\}\right\}, j=1, \ldots, m,
$$

равно $A^{\prime 1}$ ! ... $A^{\prime m}$ !. Оставшиеся элементы последовательности $\pi_{1}, \ldots, \pi_{N}$ должны быть меньше $\pi_{N_{1}-A_{m}-\ldots-A_{m+q}-q-1}$. Количество элементов, меньших $\pi_{N_{1}-A_{m}-\ldots-A_{m+q}-q-1}$, равно $N-N_{2}-1$. Остаётся из этих $N-N_{2}-1$ элементов выбрать упорядоченный набор из $N-N_{1}-N_{2}+N_{3}$ элементов, не вошедших в последовательности $\pi_{1}, \ldots, \pi_{N_{1}}$ и $\pi_{1}^{\prime}, \ldots, \pi_{N_{2}-N_{3}+1}^{\prime}$. Количество таких упорядоченных наборов равно $\frac{\left(N-N_{2}-1\right) !}{\left(N_{1}-N_{3}-1\right) !}$. В итоге получаем

$$
\begin{aligned}
\mathrm{P}\left(B_{1}^{m+n} \cap B_{m+1}^{m+q+n}\right. & \cap\{L(m+q+n+1)=N\})= \\
=C_{N-N_{1}-1}^{N_{2}-N_{3}-1} & \prod_{s=1}^{m+q} \frac{A_{s} !}{a_{s}^{1} ! \ldots a_{s}^{s} !} \prod_{t=m+q+1}^{m+q+n} \frac{A_{t}^{\prime \prime} !}{a_{t}^{m+1} ! \ldots a_{t}^{t} !} \times \\
& \times A^{m+1} ! \ldots A^{m+q+n} ! A^{\prime \prime} ! \ldots A^{\prime m} ! \frac{\left(N-N_{2}-1\right) !}{\left(N_{1}-N_{3}-1\right) !} \frac{1}{N !} .
\end{aligned}
$$

Из теорем 1 и 2 следует, что

$$
\begin{aligned}
& \mathrm{P}\left(B_{1}^{m+q} \cap B_{m+1}^{m+q+n}\right.\cap\{L(m+q+n+1)=N\})=\frac{\mathrm{P}\left(B_{1}^{m+q}\right) \mathrm{P}\left(B_{m+1}^{m+q+n}\right)}{\mathrm{P}\left(B_{m+1}^{m+q}\right)} \times \\
& \times \frac{N_{1} ! N_{2} !\left(N-N_{2}-1\right) !\left(N-N_{1}-1\right) !}{N ! N_{3} !\left(N_{1}-N_{3}-1\right) !\left(N_{2}-N_{3}-1\right) !\left(N-N_{1}-N_{2}+N_{3}\right) !} .
\end{aligned}
$$

Для суммирования (3) по $N$ используем следующее тождество.

Лемма 1. Пусть а, c,d-иелье неотрицательные числа. Тогда

$$
\sum_{b=0}^{\infty} \frac{(a+b) !(b+c) !}{b ! d !(a+b+c+d+2) !}=\frac{a !}{(a+d+1) !} \cdot \frac{c !}{(c+d+1) !} .
$$

Доказательство леммы 1.

$$
\begin{gathered}
\frac{(a+b) !(b+c) !}{b ! d !(a+b+c+d+2) !}=\left(\begin{array}{c}
a+b \\
b
\end{array}\right) \cdot \frac{a !(b+c) !}{d !(a+b+c+d+2) !}= \\
=\left(\begin{array}{c}
a+b \\
b
\end{array}\right) \cdot \frac{(a+d+1) !(b+c) !}{(a+b+c+d+2) !} \cdot \frac{a !}{d !(a+d+1) !}= \\
=\frac{a !}{d !(a+d+1) !} \cdot\left(\begin{array}{c}
a+b \\
b
\end{array}\right) B(a+d+2, b+c+1),
\end{gathered}
$$


где $B(\cdot, \cdot)$ обозначает бета-функцию.

$$
\begin{gathered}
\sum_{b=0}^{\infty}\left(\begin{array}{c}
a+b \\
b
\end{array}\right) B(a+d+2, b+c+1)=\sum_{b=0}^{\infty}\left(\begin{array}{c}
a+b \\
b
\end{array}\right) \int_{0}^{1} x^{b+c}(1-x)^{a+d+1} d x= \\
=\int_{0}^{1} x^{c}(1-x)^{a+d+1} \sum_{b=0}^{\infty}\left(\begin{array}{c}
a+b \\
b
\end{array}\right) x^{b} d x .
\end{gathered}
$$

Так как (см., например, [7; гл. II, § 8])

$$
\sum_{b=0}^{\infty}\left(\begin{array}{c}
a+b \\
b
\end{array}\right) x^{b}=(1-x)^{-(a+1)}, \quad 0<x<1,
$$

Tо

$$
\begin{aligned}
\int_{0}^{1} x^{c}(1-x)^{a+d+1} \sum_{b=0}^{\infty}\left(\begin{array}{c}
a+b \\
b
\end{array}\right) x^{b} d x & =\int_{0}^{1} x^{c}(1-x)^{a+d+1}(1-x)^{-(a+1)} d x= \\
& =\int_{0}^{1} x^{c}(1-x)^{d} d x=B(c+1, d+1)=\frac{c ! \cdot d !}{(c+d+1) !}
\end{aligned}
$$

Учитывая (4), завершаем доказательство леммы 1:

$$
\sum_{b=0}^{\infty} \frac{(a+b) ! \cdot(b+c) !}{b ! d !(a+b+c+d+2) !}=\frac{a !}{(a+d+1) !} \cdot \frac{c !}{(c+d+1) !} .
$$

Лемма доказана.

Из леммы 1 следует, что

$$
\sum_{N=N_{1}+N_{2}-N_{3}}^{\infty} \frac{N_{1} ! N_{2} !\left(N-N_{1}-1\right) !\left(N-N_{2}-1\right) !}{N ! N_{3} !\left(N_{1}-N_{3}-1\right) !\left(N_{2}-N_{3}-1\right) !\left(N-N_{1}-N_{2}+N_{3}\right) !}=1
$$

(достаточно положить $a=N_{1}-N_{3}-1, b=N-N_{1}-N_{2}+N_{3}, c=N_{2}-N_{3}-1$, $\left.d=N_{3}\right)$. Поэтому в силу $(2)$,

$$
\mathrm{P}\left(B_{1}^{m+q} \cap B_{m+1}^{m+q+n}\right)=\frac{\mathrm{P}\left(B_{1}^{m+q}\right) \cdot \mathrm{P}\left(B_{m+1}^{m+q+n}\right)}{\mathrm{P}\left(B_{m+1}^{m+q}\right)} .
$$

Так как $B_{1}^{m+q} \subset B_{m+1}^{m+q}$ и $B_{m+1}^{m+q+n} \subset B_{m+1}^{m+q}$, то приходим к равенству из теоремы 4 :

$$
\mathrm{P}\left(B_{1}^{m+q} \cap B_{m+1}^{m+q+n} \mid B_{m+1}^{m+q}\right)=\mathrm{P}\left(B_{1}^{m+q} \mid B_{m+1}^{m+q}\right) \cdot \mathrm{P}\left(B_{m+1}^{m+q+n} \mid B_{m+1}^{m+q}\right) .
$$

Если провести те же рассуждения при $q=0$ (считая $B_{m+1}^{m+q}$ достоверным событием), то получим равенство из теоремы 3 :

$$
\mathrm{P}\left(B_{1}^{m} \cap B_{m+1}^{m+n}\right)=\mathrm{P}\left(B_{1}^{m}\right) \cdot \mathrm{P}\left(B_{m+1}^{m+n}\right) .
$$

Теоремы 3 и 4 доказаны. 


\section{3. Асимптотические свойства одномерного распреде- ления $\alpha_{i}^{j}$. Доказательства теорем 5-7}

При доказательстве теорем $5,6,7$ будем считать, что $F(x)=1-e^{-x}$, поскольку распределения рекордных, межрекордных моментов и межрекордных наполнений не зависят от распределения $X_{1}$. Тогда $X_{L(n)+1}, X_{L(n)+2}, \ldots$ имеют одно и то же экспоненциальное распределение, взаимно независимы и не зависят от $X(1), \ldots, X(n)$, где $X(i)=X_{L(i)}, i \geqslant 1$.

Индукцией по $k$ с использованием того, что при $F(x)=1-e^{-x}$ условное распределение $X(k)-X(k-1)$ при условии $X(k-1)=z$ имеет функцию распределения $F(x)$ и не зависит от $z$, нетрудно доказать справедливость следующей формулы для распределения $\Delta(k)$ :

$$
\mathrm{P}(\Delta(k)>n)=\int_{0}^{\infty} \frac{x^{k-1}}{(k-1) !} e^{-x}\left(1-e^{-x}\right)^{n} d x .
$$

Применение этого интегрального представления для нахождения некоторых предельных соотношений можно найти в статье Ньютса [4].

Найдем аналогичное интегральное представление распределений межрекордных наполнений (в силу следствия 1 достаточно найти распределения $\alpha_{k}^{1}$ ).

Доказательство теоремы 5. При $k=1$

$$
\begin{gathered}
\mathrm{P}\left(\alpha_{1}^{1} \geqslant m\right)=\mathrm{P}(L(2)-L(1)-1 \geqslant m)= \\
=\mathrm{P}(L(2)>m+1)=\mathrm{P}\left(X_{1}>\max _{2 \leqslant n \leqslant m+1} X_{n}\right)=\frac{1}{m+1} .
\end{gathered}
$$

Пусть теперь $k>1$. По формуле полной вероятности по значениям $\Delta(k)$

$$
\mathrm{P}\left(\alpha_{k}^{1}=n\right)=\sum_{s=n+1}^{\infty} \mathrm{P}\left(\alpha_{k}^{1}=n, \Delta(k)=s\right) .
$$

Так как по предположению $F(x)=1-e^{-x}$, то $X(1)=X_{1}, X(2)-X(1), \ldots, X(n)-$ $X(n-1)$ - независимые случайные величины с тем же экспоненциальным распределением. Поэтому $X(k)-X(1)$ не зависит от $X_{1}$ и имеет гамма-распределение с параметром $k-1$ и плотностью $\frac{x^{k-2}}{(k-2) !} e^{-x}$. По формуле полной вероятности по значениям $X_{1}$ и $X(k)-X_{1}$

$$
\begin{gathered}
\mathrm{P}\left(\alpha_{k}^{1}=n, \Delta(k)=s\right)= \\
=\int_{0}^{\infty} \int_{0}^{\infty} \mathrm{P}\left\{\alpha_{k}^{1}=n, \Delta(k)=s \mid X_{1}=y, X(k)-X_{1}=x\right\} d F(y) d F_{X(k)-X(1)}(x) .
\end{gathered}
$$


По формуле полной вероятности по значениям $L(k)$ получаем:

$$
\begin{aligned}
& \mathrm{P}\left\{\alpha_{k}^{1}=n, \Delta(k)=s \mid X_{1}=y, X(k)=x+y\right\}= \\
&=\sum_{l=k}^{\infty} \mathrm{P}\left(\alpha_{k}^{1}=n, \Delta(k)=s \mid L(k)=l, X_{1}=y, X(k)=x+y\right) \times \\
& \times \mathrm{P}\left(L(k)=l \mid X_{1}=y, X(k)=x+y\right) .
\end{aligned}
$$

Далее,

$$
\begin{gathered}
\mathrm{P}\left\{\alpha_{k}^{1}=n, \Delta(k)=s \mid L(k)=l, X_{1}=y, X(k)=x+y\right\}= \\
=\mathrm{P}\left\{\sum_{m=1}^{s-1} I\left\{X_{l+m}<y\right\}=n, \max _{l<m<l+s} X_{m}<x+y, X_{l+s}>x+y \mid X_{1}=y,\right. \\
\left.X_{l}=x+y>\max _{1 \leqslant m<l} X_{m}, L(k)=l\right\} .
\end{gathered}
$$

Условие зависит только от случайных величин $X_{1}, \ldots, X_{l}$, а событие внутри вероятности только от случайных величин $X_{l+1}, \ldots, X_{l+s}$, поэтому условие можно убрать $(x, y-$ фиксированные числа). Значит,

$$
\begin{aligned}
& \mathrm{P}\left\{\alpha_{k}^{1}=n, \Delta(k)=s \mid L(k)=l, X_{1}=y, X(k)=x+y\right\}= \\
& =\left(\begin{array}{c}
s-1 \\
n
\end{array}\right) F^{n}(y)(F(x+y)-F(y))^{s-n-1}(1-F(x+y)) .
\end{aligned}
$$

Возвращаясь к (7), находим, что

$$
\begin{gathered}
\mathrm{P}\left\{\alpha_{k}^{1}=n, \Delta(k)=s \mid X_{1}=y, X(k)=x+y\right\}= \\
=\sum_{l=k}^{\infty}\left(\begin{array}{c}
s-1 \\
n
\end{array}\right) F^{n}(y)(F(x+y)-F(y))^{s-n-1}(1-F(x+y)) \times \\
\times \mathrm{P}\left(L(k)=l \mid X_{1}=y, X(k)=x+y\right)= \\
=\left(\begin{array}{c}
s-1 \\
n
\end{array}\right) F^{n}(y)(F(x+y)-F(y))^{s-n-1}(1-F(x+y)) .
\end{gathered}
$$

Суммируя (6) по $s$, пользуясь (8) и (5), получаем, что

$$
\mathrm{P}\left(\alpha_{k}^{1}=n\right)=\int_{0}^{\infty} \int_{0}^{\infty} \frac{F^{n}(y)}{(1+F(y)-F(x+y))^{n+1}} \cdot \bar{F}(x+y) d F(y) d F_{X(k)-X(1)}(x) .
$$

Отсюда и из того, что $d F_{X(k)-X(1)}(x)=\frac{x^{k-2}}{(k-2) !} e^{-x} d x$, следует утверждение теоремы 5 :

$$
\mathrm{P}\left(\alpha_{k}^{1} \geqslant m\right)=\sum_{n=m}^{\infty} \mathrm{P}\left(\alpha_{k}^{1}=n\right)=\int_{0}^{\infty} \frac{x^{k-2}}{(k-2) !} e^{-x} \int_{0}^{\infty}\left[\frac{1-e^{-y}}{1+e^{-x-y}-e^{-y}}\right]^{m} d F(y) d x .
$$

Теорема доказана.

3 Дискретная математика, т. 27 № 3 
Перейдем к доказательству теоремы 6. В [2] показано, что $\ln L(k)$ и $\ln \Delta(k)$ асимптотически близки, а именно, доказано, что с вероятностью единица выполняется соотношение

$$
\limsup _{k \rightarrow \infty}\left|\frac{\ln L(k)-\ln \Delta(k)}{\ln k}\right|=1 .
$$

Откуда следуют аналогичные предельным соотношениям для $L(k)$, доказанным в [2], предельные соотношения для случайных величин $\Delta(k)$ (ЦПТ, УЗБЧ, закон повторного логарифма):

$$
\begin{gathered}
\frac{\ln \Delta(k)-k}{\sqrt{k}} \stackrel{D}{\underset{k \rightarrow \infty}{\longrightarrow}} N(0,1), \quad \lim _{k \rightarrow \infty} \frac{\ln \Delta(k)}{k} \stackrel{\text { п.н. }}{=} 1, \\
\limsup _{k \rightarrow \infty} \frac{\ln \Delta(k)-k}{\sqrt{2 k \ln \ln k}} \stackrel{\text { п.н. }}{=} 1, \quad \liminf _{k \rightarrow \infty} \frac{\ln \Delta(k)-k}{\sqrt{2 k \ln \ln k}} \stackrel{\text { п.н. }}{=}-1 .
\end{gathered}
$$

Теорема 8. С вероятностью единица справедливо следующее предельное соотношение:

$$
\limsup _{k \rightarrow \infty}\left|\frac{\ln \alpha_{k}^{1}-\ln \Delta(k)}{\ln k}\right| \leqslant 1
$$

Доказательства теоремы 8 и теоремы 7 используют представление межрекордных наполнений в виде суммы случайного числа индикаторов:

$$
\alpha_{k}^{1}=\xi_{1}^{k}\left(X_{1}\right)+\ldots+\xi_{\Delta(k)-1}^{k}\left(X_{1}\right),
$$

где $\xi_{i}^{k}(y)=I\left\{X_{L(k)+i}<y\right\}, i \geqslant 1$. Если $F(x)=1-e^{-x}$, то случайные величины $X_{1}$, $\left\{\xi_{i}^{k}(y), i \geqslant 1\right\}$ независимы в совокупности при фиксированных $k$ и $y$, и $\mathrm{P}\left\{\xi_{i}^{k}(y)=\right.$ $1\}=F(y)$. Таким образом, мы представили межрекордные наполнения вида $\alpha_{k}^{1}$ как сумму случайного числа случайных величин, условно независимых при фиксированном значении первого рекорда (межрекордные наполнения вида $\alpha_{k}^{j}, j>1$ можно представить в виде суммы индикаторов вида $\left.I\left\{X(j-1)<X_{L(k)+i}<X(j)\right\}\right)$.

Доказательство теоремы 8.

Лемма 2. Для любого $k>2$ выполнено неравенство

$$
\mathrm{P}\left\{\frac{\alpha_{k}^{1}}{\Delta(k)} \leqslant \frac{1}{k \ln ^{2} k}\right\} \leqslant \frac{C}{k \ln ^{2} k},
$$

где константа $C$ не зависит от $k$.

Выведем сначала из леммы 2 теорему 8, а затем докажем лемму. Воспользуемся методом, представленным в [2] в доказательстве соотношения (9). Из леммы 2 следует, что ряд $\sum_{k=1}^{\infty} \mathrm{P}\left(\frac{\alpha_{k}^{1}}{\Delta(k)} \leqslant \frac{1}{k \ln ^{2} k}\right) \quad$ сходится. Согласно лемме Бореля-Кантелли это означает, что

$$
\mathrm{P}\left(\exists N \in \mathbb{N}, \frac{\alpha_{k}^{1}}{\Delta(k)}>\frac{1}{k \ln ^{2} k} \text { для всех натуральных } k>N\right)=1 .
$$


Отсюда следует, что соотношение $\liminf _{n \rightarrow \infty} \frac{\ln \alpha_{k}^{1}-\ln \Delta(k)}{\ln k} \geqslant-1$ выполнено с вероятностью 1. Учитывая, что $\alpha_{k}^{1}<\Delta(k)$, получим, что с вероятностью 1 выполнено неравенство $\limsup _{n \rightarrow \infty}\left|\frac{\ln \alpha_{k}^{1}-\ln \Delta(k)}{\ln k}\right| \leqslant 1$.

Теорема 8 доказана.

Доказательство леммы 2. Используем формулу полной вероятности по значениям $\Delta(k)$ и выделим сумму вероятностей событий с $\Delta(k) \leqslant\left(k \ln ^{2} k\right)^{3}$ и сумму остальных членов ряда:

$$
\begin{gathered}
\mathrm{P}\left(\frac{\alpha_{k}^{1}}{\Delta(k)} \leqslant \frac{1}{2 k \ln ^{2} k}\right)=\sum_{n=1}^{\infty} \mathrm{P}\left(\frac{\alpha_{k}^{1}}{\Delta(k)} \leqslant \frac{1}{2 k \ln ^{2} k}, \Delta(k)=n\right) \leqslant \\
\leqslant \mathrm{P}\left(\Delta(k) \leqslant\left(k \ln ^{2} k\right)^{3}\right)+\sum_{n=\left(k \ln ^{2} k\right)^{3}+1}^{\infty} \mathrm{P}\left(\frac{\alpha_{k}^{1}}{\Delta(k)} \leqslant \frac{1}{2 k \ln ^{2} k}, \Delta(k)=n\right) .
\end{gathered}
$$

Докажем, что оба слагаемых не превосходят $\frac{C}{k \ln ^{2} k}$ для некоторой константы $C$, не зависящей от $k$.

Межрекордное наполнение $\alpha_{k}^{1}$ представляется суммой $\Delta(k)-1$ слагаемых (представление (10)), поэтому для оценивания второго слагаемого в (11) естественно перейти от величины $\frac{\alpha_{k}^{1}}{\Delta(k)}$ к величине $\frac{\alpha_{k}^{1}}{\Delta(k)-1}$, используя неравенство $\frac{\Delta(k)}{\Delta(k)-1} \leqslant 2$ при $\Delta(k)>1$ :

$$
\begin{aligned}
& \sum_{n=\left(k \ln ^{2} k\right)^{3}+1}^{\infty} \mathrm{P}\left(\frac{\alpha_{k}^{1}}{\Delta(k)} \leqslant \frac{1}{2 k \ln ^{2} k}, \Delta(k)=n\right) \leqslant \\
\leqslant & \sum_{n=\left(k \ln ^{2} k\right)^{3}}^{\infty} \mathrm{P}\left(\frac{\alpha_{k}^{1}}{\Delta(k)-1} \leqslant \frac{1}{k \ln ^{2} k}, \Delta(k)=n+1\right) .
\end{aligned}
$$

Так как $\alpha_{k}^{1}$ представляется суммой условно независимых слагаемых при условии фиксированного значения первого рекорда, будем фиксировать первый рекорд. По формуле полной вероятности, рассматривая отдельно значения $X_{1}$, не превосходящие $F^{-1}\left(\frac{2}{k \ln ^{2} k}\right)$ и остальные значения $X_{1}$, оцениваем сумму (12):

$$
\begin{gathered}
\sum_{n=\left(k \ln ^{2} k\right)^{3}}^{\infty} \mathrm{P}\left\{\frac{\alpha_{k}^{1}}{\Delta(k)-1} \leqslant \frac{1}{k \ln ^{2} k}, \Delta(k)=n+1\right\} \leqslant \\
\leqslant \frac{2}{k \ln ^{2} k}+\sum_{n=\left(k \ln ^{2} k\right)^{3}{ }_{F}^{-1}\left(\frac{2}{k \ln ^{2} k}\right)}^{\infty} \int^{\infty} \mathrm{P}\left(\frac{\alpha_{k}^{1}}{\Delta(k)-1} \leqslant \frac{1}{k \ln ^{2} k}, \Delta(k)=n+1 \mid X_{1}=y\right) d F(y) .
\end{gathered}
$$


Так как интегрирование в (13) идет только по таким $y$, что $F(y) \geqslant \frac{2}{k \ln ^{2} k}$, а $X_{1}$ и $\left\{\xi_{i}^{k}(y), i \geqslant 1\right\}$ независимы, получаем $\left(\mathrm{M} \xi_{1}^{k}(y)=F(y)\right)$ :

$$
\begin{gathered}
\mathrm{P}\left(\frac{\alpha_{k}^{1}}{\Delta(k)-1} \leqslant \frac{1}{k \ln ^{2} k}, \Delta(k)=n+1 \mid X_{1}=y\right) \leqslant \\
\leqslant \mathrm{P}\left(\frac{\xi_{1}^{k}(y)+\ldots+\xi_{n}^{k}(y)}{n}-\mathrm{M} \xi_{1}^{k}(y) \leqslant \frac{1}{k \ln ^{2} k}-F(y)\right) \leqslant \\
\leqslant \mathrm{P}\left(\frac{\xi_{1}^{k}(y)+\ldots+\xi_{n}^{k}(y)}{n}-\mathrm{M} \xi_{1}^{k}(y) \leqslant-\frac{1}{k \ln ^{2} k}\right) .
\end{gathered}
$$

Случайные величины $\xi_{1}^{k}(y), \xi_{2}^{k}(y), \xi_{3}^{k}(y) \ldots$ независимы и имеют распределение Бернулли. По неравенству Хефдинга [5; §6 гл. І, стр. 81, формула (41)] справедливо неравенство

$$
\mathrm{P}\left(\frac{\xi_{1}^{k}(y)+\ldots+\xi_{n}^{k}(y)}{n}-\mathrm{M} \xi_{1}^{k}(y) \leqslant-\frac{1}{k \ln ^{2} k}\right) \leqslant \exp \left\{-2 n\left(\frac{1}{k \ln ^{2} k}\right)^{2}\right\}
$$

Отсюда после суммирования геометрической прогрессии находим:

$$
\begin{gathered}
\sum_{n=\left(k \ln ^{2} k\right)^{3}}^{\infty} \mathrm{P}\left(\frac{\xi_{1}^{k}(y)+\ldots+\xi_{n}^{k}(y)}{n}-\mathrm{M} \xi_{1}^{k}(y) \leqslant \frac{1}{k \ln ^{2} k}-F(y)\right) \leqslant \\
\leqslant e^{-2 k \ln ^{2} k} \frac{1}{1-e^{-2\left(\frac{1}{k \ln ^{2} k}\right)^{2}}} \leqslant \frac{C_{1}}{k \ln ^{2} k},
\end{gathered}
$$

где $C_{1}$ - константа, не зависящая от $k$. Такая константа существует, поскольку

$$
k \ln ^{2} k \cdot e^{-2 k \ln ^{2} k} \frac{1}{1-e^{-2\left(\frac{1}{k \ln ^{2} k}\right)^{2}}} \sim \frac{1}{2}\left(k \ln ^{2} k\right)^{3} \cdot e^{-2 k \ln ^{2} k} \rightarrow 0 \quad \text { при } k \rightarrow \infty .
$$

Осталось показать, что $\mathrm{P}\left(\Delta(k) \leqslant\left(k \ln ^{2} k\right)^{3}\right) \leqslant \frac{C_{2}}{k \ln ^{2} k}$, где $C_{2}$ - константа, не зависящая от $k$. Для этого достаточно показать, что $k \ln ^{2} k \mathrm{P}\left(\Delta(k) \leqslant\left(k \ln ^{2} k\right)^{3}\right) \underset{k \rightarrow \infty}{\longrightarrow} 0$. Функция распределения $\Delta(k)$ представляется в интегральном виде так [4]:

$$
\begin{gathered}
\mathrm{P}(\Delta(k) \leqslant z)= \\
=1-\int_{0}^{\infty} \frac{x^{k-1}}{(k-1) !} e^{-x}\left[1-e^{-x}\right]^{z} d x=\int_{0}^{\infty} \frac{x^{k-1}}{(k-1) !} e^{-x}\left(1-\left[1-e^{-x}\right]^{z}\right) d x .
\end{gathered}
$$


Полагая здесь $z=\left(k \ln ^{2} k\right)^{3}$ и используя неравенство Бернулли $\left[1-e^{-x}\right]^{z} \geqslant 1-z e^{-x}$, получаем:

$$
\begin{gathered}
k \ln ^{2} k \mathrm{P}\left(\Delta(k) \leqslant\left(k \ln ^{2} k\right)^{3}\right)= \\
=\int_{0}^{\infty} k \ln ^{2} k \frac{x^{k-1}}{(k-1) !} e^{-x}\left(1-\left[1-e^{-x}\right]^{\left(k \ln ^{2} k\right)^{3}}\right) d x \leqslant \\
\leqslant \int_{0}^{\infty}\left(k \ln ^{2} k\right)^{4} \frac{x^{k-1}}{(k-1) !} e^{-2 x} d x=\int_{0}^{\infty} \frac{\left(k \ln ^{2} k\right)^{4}}{2^{k}} \frac{(2 x)^{k-1}}{(k-1) !} e^{-2 x} d(2 x)= \\
=\int_{0}^{\infty} \frac{\left(k \ln ^{2} k\right)^{4}}{2^{k}} \frac{y^{k-1}}{(k-1) !} e^{-y} d y=\frac{\left(k \ln ^{2} k\right)^{4}}{2^{k}} .
\end{gathered}
$$

Поскольку $2^{-k}\left(k \ln ^{2} k\right)^{4} \underset{k \rightarrow \infty}{\longrightarrow} 0$, это завершает доказательство леммы.

Из теоремы 8 следуют предельные соотношения, сформулированные в теореме 6 (асимптотическая нормальность, усиленный закон больших чисел, закон повторного логарифма):

$$
\begin{gathered}
\frac{\ln \alpha_{k}^{1}-k}{\sqrt{k}} \underset{k \rightarrow \infty}{\stackrel{D}{\longrightarrow}} N(0,1), \quad \lim _{k \rightarrow \infty} \frac{\ln \alpha_{k}^{1}}{k} \stackrel{\text { п.н. }}{=} 1, \\
\limsup _{k \rightarrow \infty} \frac{\ln \alpha_{k}^{1}-k}{\sqrt{2 k \ln \ln k}} \stackrel{\text { п.н. }}{=} 1, \quad \liminf _{k \rightarrow \infty} \frac{\ln \alpha_{k}^{1}-k}{\sqrt{2 k \ln \ln k}} \stackrel{\text { п.н. }}{=}-1 .
\end{gathered}
$$

Действительно, для доказательства выполнения усиленного закона больших чисел для $\alpha_{k}^{1}$ заметим, что всегда выполнено неравенство

$$
\left|\frac{\ln \alpha_{k}^{1}}{k}-1\right| \leqslant\left|\frac{\ln \alpha_{k}^{1}-\ln \Delta(k)}{k}\right|+\left|\frac{\ln \Delta(k)}{k}-1\right| .
$$

Теперь, если воспользоваться теоремой 8, УЗБЧ для $\Delta(k)$ и неравенством $(15)$, получим, что $\lim _{k \rightarrow \infty} \frac{\ln \alpha_{k}^{1}}{k} \stackrel{\text { п.н. }}{=} 1$.

Аналогично доказывается закон повторного логарифма для $\alpha_{k}^{1}$.

Для доказательства асимптотической нормальности воспользуемся теоремой непрерывности (см. [6; стр. 36]).

Теорема непрерывности. Пусть $\eta_{n} u \eta-$ случайные векторь из $\mathbb{R}^{s}$. Если $\eta_{n} \underset{n \rightarrow \infty}{\stackrel{D}{\longrightarrow}} \eta u H(t), t \in \mathbb{R}^{s}$, есть непрерьвная функиия из $\mathbb{R}^{s}$ в $\mathbb{R}^{k}$, mо $H\left(\eta_{n}\right) \underset{n \rightarrow \infty}{\longrightarrow}$ $H(\eta)$.

В частности, из теоремы непрерывности следует, что если $X_{n}, Y_{n}(n \in \mathbb{R})-$ случайные величины и $X_{n} \underset{n \rightarrow \infty}{\stackrel{D}{\longrightarrow}} X, Y_{n} \underset{n \rightarrow \infty}{\stackrel{\mathrm{P}}{\longrightarrow}} 0$, то $X_{n}+Y_{n} \underset{n \rightarrow \infty}{\stackrel{D}{\longrightarrow}} X$.

Из ЦПТ для $\Delta(k)$ имеем

$$
\frac{\ln \Delta(k)-k}{\sqrt{k}} \underset{k \rightarrow \infty}{\stackrel{D}{\longrightarrow}} N(0,1) .
$$


По теореме 8

$$
\frac{\ln \alpha_{k}^{1}-\ln \Delta(k)}{\sqrt{k}} \underset{k \rightarrow \infty}{\stackrel{\text { п.н }}{\longrightarrow}} 0,
$$

а значит, имеет место сходимость по вероятности

$$
\frac{\ln \alpha_{k}^{1}-\ln \Delta(k)}{\sqrt{k}} \underset{k \rightarrow \infty}{\stackrel{\mathrm{P}}{\longrightarrow}} 0 .
$$

Отсюда, по частному случаю теоремы непрерывности, описанному выше, получаем

$$
\frac{\ln \alpha_{k}^{1}-k}{\sqrt{k}} \underset{k \rightarrow \infty}{\stackrel{D}{\longrightarrow}} N(0,1) \text {. }
$$

Теорема 6 доказана.

Предельное распределение отношения $\frac{\Delta(k)}{L(k+1)}$ является равномерным распределением на отрезке $[0,1]$. Действительно, в [3; стр. 17] было найдено предельное распределение отношения $\frac{L(k+1)}{L(k)}$ :

$$
\lim _{k \rightarrow \infty} \mathrm{P}\left(\frac{L(k+1)}{L(k)}>x\right)=\frac{1}{x}, \quad x \geqslant 1 .
$$

Значит, для любого $0<x<1$

$$
\begin{aligned}
\mathrm{P}\left(\frac{\Delta(k)}{L(k+1)}>x\right)=\mathrm{P}\left(1-\frac{L(k)}{L(k+1)}>x\right) & = \\
& =\mathrm{P}\left(\frac{L(k+1)}{L(k)}>\frac{1}{1-x}\right) \underset{k \rightarrow \infty}{\longrightarrow} 1-x .
\end{aligned}
$$

Очевидно, что $\mathrm{P}\left(\frac{\Delta(k)}{L(k+1)}>0\right)=1$ и $\mathrm{P}\left(\frac{\Delta(k)}{L(k+1)}>1\right)=0$. Следовательно,

$$
\lim _{k \rightarrow \infty} \mathrm{P}\left(\frac{\Delta(k)}{L(k+1)}>x\right)=1-x, \quad 0 \leqslant x \leqslant 1 .
$$

Доказательство теоремы 7 . Покажем, что предельное распределение отношения $\frac{\alpha_{k}^{1}}{\Delta(k)}$ тоже является равномерным на отрезке $[0,1]$.

Достаточно показать, что $\mathrm{P}\left\{\frac{\alpha_{k}^{1}}{\Delta(k)-1}>x\right\} \underset{k \rightarrow \infty}{\longrightarrow} 1-x, 0 \leqslant x \leqslant 1$.

По формуле полной вероятности по $X_{1}$

$$
\mathrm{P}\left\{\frac{\alpha_{k}^{1}}{\Delta(k)-1}>x\right\}=\int_{0}^{\infty} \mathrm{P}\left\{\frac{\alpha_{k}^{1}}{\Delta(k)-1}>x \mid X_{1}=y\right\} d F(y) .
$$

Далее, рассмотрим вероятностную меру $\mathrm{P}_{\left\{X_{1}=y\right\}}(A)=\mathrm{P}\left(A \mid X_{1}=y\right)$, где $y$ - фиксированное число, а $A$ - некоторое событие. Вспоминая представление (10), получаем равенство

$$
\mathrm{P}\left\{\frac{\alpha_{k}^{1}}{\Delta(k)-1} \mid X_{1}=y\right\}=\mathrm{P}\left\{\frac{1}{\Delta(k)-1} \sum_{i=1}^{\Delta(k)-1} \xi_{i}^{k}(y) \mid X_{1}=y\right\}
$$


Последовательность случайных величин $\frac{1}{\Delta(k)-1} \sum_{i=1}^{\Delta(k)-1} \xi_{i}^{k}(y)$ при $k \in \mathbb{N}$ сходится по вероятностной мере $P_{\left\{X_{1}=y\right\}}$, как это следует из леммы 3.

Лемма 3. Пусть для любого $n \in \mathbb{N}$ случайные величины $X_{n 1}, X_{n 2}, \ldots$ независимы и имеют одно и то же распределение Бернулли, $\mathrm{M} X_{n 1}=\mathrm{M} X_{11}$. Пусть $\left\{M_{n}\right\}$

- последовательность таких целых неотрицательных случайных величин, что $\mathrm{P}\left(M_{n} \leqslant m\right) \underset{n \rightarrow \infty}{\longrightarrow} 0$ для любого $m \in \mathbb{N}$. Тогда

$$
\frac{X_{n 1}+X_{n 2}+\ldots+X_{n M_{n}}}{M_{n}} \underset{n \rightarrow \infty}{\stackrel{\mathrm{P}}{\longrightarrow}} \mathrm{M} X_{11}
$$

(при $M_{n}=0$ считаем дробъ равной 0$)$.

Доказательство леммы 3. Фиксируем произвольные $\varepsilon>0$ и $\delta>0$.

По формуле полной вероятности для $M_{n}$

$$
\begin{gathered}
\mathrm{P}\left\{\left|\frac{X_{n 1}+X_{n 2}+\ldots+X_{n M_{n}}}{M_{n}}-\mathrm{M} X_{n 1}\right|>\varepsilon\right\}= \\
=\sum_{s=1}^{\infty} \mathrm{P}\left\{\left|\frac{X_{n 1}+X_{n 2}+\ldots+X_{n s}}{s}-\mathrm{M} X_{11}\right|>\varepsilon, M_{n}=s\right\} .
\end{gathered}
$$

По неравенству Хефдинга [5; стр. 81, формула (42)]

$$
\mathrm{P}\left\{\left|\frac{X_{n 1}+X_{n 2}+\ldots+X_{n s}}{s}-\mathrm{M} X_{11}\right|>\varepsilon\right\} \leqslant 2 e^{-2 s \varepsilon^{2}} \text {. }
$$

Значит, ряд $\sum_{s=1}^{\infty} \mathrm{P}\left\{\left|\frac{X_{n 1}+X_{n 2}+\ldots+X_{n s}}{s}-\mathrm{M} X_{11}\right|>\varepsilon\right\}$ сходится, причем существует такое $m \in \mathbb{N}$, что для любого $n \in \mathbb{N}$ выполнено неравенство

$$
\sum_{s=m+1}^{\infty} \mathrm{P}\left\{\left|\frac{X_{n 1}+X_{n 2}+\ldots+X_{n s}}{s}-\mathrm{M} X_{11}\right|>\varepsilon\right\}<\frac{\delta}{2} .
$$

Также существует такое $N \in \mathbb{N}$, что $\mathrm{P}\left(M_{n} \leqslant m\right)<\frac{\delta}{2}$ при всех $n>N$. Поэтому при любом $n>N$

$$
\begin{gathered}
\mathrm{P}\left\{\left|\frac{X_{n 1}+X_{n 2}+\ldots+X_{n M_{n}}}{M_{n}}-\mathrm{M} X_{11}\right|>\varepsilon\right\} \leqslant \\
\leqslant \mathrm{P}\left(M_{n} \leqslant m\right)+\sum_{s=m+1}^{\infty} \mathrm{P}\left\{\left|\frac{X_{n 1}+X_{n 2}+\ldots+X_{n s}}{s}-\mathrm{M} X_{11}\right|>\varepsilon\right\}<\delta .
\end{gathered}
$$

Лемма 3 доказана.

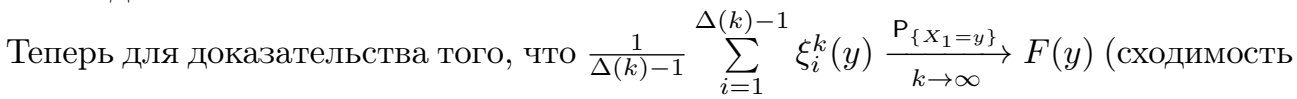
по вероятностной мере $\mathrm{P}\left(A \mid X_{1}=y\right)$ при фиксированном $\left.y\right)$, согласно лемме 3 достаточно показать, что

$$
\mathrm{P}\left\{\Delta(k)-1 \leqslant m \mid X_{1}=y\right\} \underset{k \rightarrow \infty}{\longrightarrow} 0 .
$$


По формуле полной вероятности по $X(k)-X_{1}$

$$
\begin{gathered}
\mathrm{P}\left\{\Delta(k)-1 \leqslant m \mid X_{1}=y\right\}= \\
=\int_{0}^{\infty} \mathrm{P}\left\{\Delta(k)-1 \leqslant m \mid X_{1}=y, X(k)-X_{1}=x\right\} d F_{X(k)-X_{1}}(x)= \\
=\int_{0}^{\infty} \frac{x^{k-2}}{(k-2) !} e^{-x}\left(1-\left(1-e^{-x}\right)^{m+1}\right) d x=\mathrm{P}\{\Delta(k-1) \leqslant m+1\} \underset{k \rightarrow \infty}{\longrightarrow} 0 .
\end{gathered}
$$

Покажем, что $\mathrm{P}\left\{\frac{\alpha_{k}^{1}}{\Delta(k)-1}>x \mid X_{1}=y\right\} \underset{k \rightarrow \infty}{\longrightarrow} I\{x<F(y)\}(0 \leqslant x \leqslant 1)$ и что сходимость равномерна по $y \in\left(0, F^{-1}(x)-\varepsilon\right) \bigcup\left(F^{-1}(x)+\varepsilon, \infty\right)$ для любого малого $\varepsilon>0$.

Действительно, вспомнив доказательство леммы 3, увидим, что для любого малого $\varepsilon>0$ и любого $\delta>0$ существует такое натуральное $N$, не зависящее от $y$, что для любого $y$ и для всех $k>N$

$$
\mathrm{P}\left\{\left|\frac{\alpha_{k}^{1}}{\Delta(k)-1}-F(y)\right|>\varepsilon \mid X_{1}=y\right\}<\delta .
$$

Значит, взяв $y \in\left(0, F^{-1}(x-\varepsilon)\right) \bigcup\left(F^{-1}(x+\varepsilon), \infty\right)$, получим сходимость

$$
\mathrm{P}\left\{\frac{\alpha_{k}^{1}}{\Delta(k)-1}>x \mid X_{1}=y\right\} \underset{k \rightarrow \infty}{\longrightarrow} I\{x<F(y)\}
$$

которая является равномерной на данном промежутке. Следовательно,

$$
\lim _{k \rightarrow \infty} \mathrm{P}\left\{\frac{\alpha_{k}^{1}}{\Delta(k)-1}>x\right\}=\int_{0}^{\infty} I\{x<F(y)\} d F(y)=1-x, \quad 0 \leqslant x \leqslant 1 .
$$

Статья использует материалы дипломных работ [8], [9]. Авторы признательны своему научному руководителю Василию Васильевичу Козлову за постановку задачи и постоянное внимание, а также Андрею Михайловичу Зубкову за обсуждение задачи и ценные замечания по работе.

\section{Список литературы}

1. Невзоров В.Б., Рекордъ. Математическая теория, Москва, Фазис, 2000.

2. Галамбош Я., Асимптотическая теория экстремальных порядковых статистик, М.: Наука, 1984, $304 \mathrm{c}$.

3. Tata M.N., "On outstanding values in a sequence of random variables", Z. Wahrscheinlichkeitheor. verw. Geb., 12:1 (1969), 9-20.

4. Neuts M.F., "Waiting times between record observations", J. Appl. Probab., 4:1 (1967), 206-208.

5. Ширяев А.Н., Вероятность-1, Москва, Издательство МЦНМО, 2007, 81 с. 
6. Боровков А.А., Математическая статистика, СПб.: Издательство "Лань", 2010, 36 с.

7. Феллер В., Введение в теорию вероятностей и её приложения, в 2-х томах, m.1, Москва: "Мир", 1984, 528 с.

8. Пасынков Н.Ю., Распределения в рекордных моделях, Дипломная работа, мех-мат МГУ, кафедра теории вероятностей, 2014г..

9. Орлов О.П., Предельные теоремы для порядковых распределений в межрекордных моментах, Дипломная работа, мех-мат МГУ, кафедра теории вероятностей, 2014г..

Статья поступила 12.01.2015. 